\title{
Phénoménologie des idéalités et historicité de la mathesis
}

Dominique Pradelle

\section{(2) OpenEdition}

\section{Journals}

Édition électronique

URL : http://journals.openedition.org/alter/449

DOI : $10.4000 /$ alter.449

ISSN : 2558-7927

Éditeur :

Association ALTER, Archives Husserl (CNRS-UMR 8547)

\section{Édition imprimée}

Date de publication : 3 décembre 2017

Pagination : 33-57

ISBN : 978-2-9550449-3-3

ISSN : 1249-8947

\section{Référence électronique}

Dominique Pradelle, "Phénoménologie des idéalités et historicité de la mathesis », Alter [En ligne], 25 | 2017, mis en ligne le 01 décembre 2018, consulté le 19 avril 2019. URL : http:// journals.openedition.org/alter/449; DOI : 10.4000/alter.449 


\section{PHÉNOMÉNOLOGIE DES IDÉALITES ET HISTORICITÉ DE LA MATHESIS}

Dominique Pradelle

« Je dirai quelque jour vos naissances latentes »

Rimbaud, «Voyelles »

«A présent l'inflexion éternelle des moments et l'infini des mathématiques me chassent par ce monde»

Rimbaud, Illuminations, « Guerre »

\section{Difficultés d'une eidétique de l'historicité}

Toute réflexion phénoménologique sur la structure et les modalités essentielles de l'historicité se heurte à une difficulté essentielle : comment atteindre l'invariant eidétique de toute historicité en général, quels qu'en soient le domaine et le niveau d'application ? En effet, l'application de la méthode de variation eidétique à l'histoire pose le même problème que toute variation en général, mais à un degré de difficulté accru. Toute variation, on le sait, part d'un exemple pris pour fil conducteur, puis procède à une production de variantes analogues, et finit par ressaisir l'invariant eidétique en lequel se recouvrent les différentes variantes ainsi engendrées ${ }^{1}$. Or, cela entraîne une première difficulté, liée au choix de l'orientation thématique qui détermine le point de vue depuis lequel peut s'effectuer la production des variantes : à partir d'une nuance de rouge, je puis en effet déployer la variation selon divers degrés d'analogie, et dégager ou bien l'eidos du rouge en général, ou bien celui de la couleur en général, voire de la qualité sensible en général ${ }^{2}$; $1^{\prime}$ exemple ne suffit

${ }^{1}$ E. Husserl, Erfahrung und Urteil, § 87a, Hambourg, Glaassen \& Goverts, 1954 (reed. F. Meiner, 1999), p. 410-411 (trad. D. Souche, Expérience et jugement, Paris, PUF, 1970, p. 413-414).

2 Ibid., § 92, p. 433 (trad., p. 435). 
donc pas à déterminer de façon univoque le niveau d'analogie des variantes, lequel demeure tributaire d'une orientation thématique initiale, d'une visée préalable de généralité qui vectorise souterrainement la variation.

Mais il existe une seconde difficulté, tout à fait inverse, qui fait obstacle au dégagement de l'eidos de l'historicité : la variation eidétique peut en effet, en ce cas, demeurer enchaînée par une double factualité du point de départ de la variation. D'une part, la factualité de la datation ou du moment envisagé, de la période historique que l'on détache au sein de l'histoire globale: parviendra-t-on, à partir d'une époque de la Grèce antique, de l'Antiquité romaine, du Moyen Âge ou de l'âge classique, aux mêmes traits généraux de l'historicité en général, sans que ces derniers soient en rien affectés par le visage particulier de l'époque considérée? D’autre part, celle du niveau thématique choisi au sein de l'histoire culturelle : parviendra-t-on aux mêmes invariants eidétiques selon que l'on envisage l'histoire politique, militaire, celle des sciences ou de telle science particulière, celle des religions ou de telle religion particulière, celle des arts ou de tel art particulier, sans que ce choix thématique influe en rien sur la nature des traits eidétiques dégagés ? Cette factualité avait sur le tard conduit Husserl à ne pas thématiser l'historicité en général, mais à distinguer des «niveaux de l'historicité » (Stufen der Geschichtlichkeit) - c'est-à-dire à reconnaître le caractère polymorphe des structures de l'histoire et de ses modes de constitution selon le type de praxis et de tradition culturelles qui est envisagé ${ }^{3}$. Deux questions sont ainsi ouvertes. Tout d'abord, est-il d'emblée évident que l'eidos d'historicité soit invariant et possède des structures universelles, communes à tout segment d'histoire, qui se laissent dégager à l'identique à partir d'un quelconque moment ou période de l'histoire ? En outre, est-il d'emblée évident que cet eidos possède les mêmes structures eidétiques pour tous les niveaux d'historicité envisageables - c'est-à-dire quelle que soit la discipline, l'activité ou le type de fait culturel que l'on adopte pour paradigme?

Bref, le choix de l'exemple de départ et l'orientation thématique demeurentelles sans incidence sur les traits eidétiques qui seront ressaisis au terme de la variation?

\footnotetext{
${ }^{3}$ E. Husserl, Die Krisis der europäischen Wissenschaften und die transzendentale Phänomenologie, Beilage XXVI zu § 73, Hua VI, La Haye, Martinus Nijhoff, 1954, p. 502-503 (trad. G. Granel, La crise des sciences européennes et la phénoménologie transcendantale, Paris, Gallimard, 1976, p. 556557).
} 
De fait, au début de «L'origine de la géométrie », Husserl met l'accent sur le caractère exemplaire ou paradigmatique de ses considérations sur l'histoire de la géométrie :

nos considérations conduiront vers les plus profonds problèmes de sens, problèmes de la science et de l'histoire de la science en général, et même finalement d'une histoire universelle en général ; si bien que nos problèmes et explicitations touchant la géométrie galiléenne détiennent une signification exemplaire [exemplarische Bedeutung] $]^{4}$.

Les considérations de Husserl sur la géométrie ont une portée généralisante : il prétend passer de l'eidos spécifique de l'historicité de la géométrie à l'eidos générique de l'historicité de toute science en général, puis de l'histoire universelle qui englobe tous les niveaux de l'historicité ; en d'autres termes, ressaisir, sur le paradigme qu'est l'histoire de la géométrie comme discipline théorétique, des traits eidétiques qui seront a priori transposables à toute forme d'historicité en général - comme si l'eidos de l'historicité se donnait à déchiffrer sur tout exemple de discipline ou de tradition historique.

Or, ne serait-ce pas plutôt l'inverse? N'est-ce pas plutôt parce que, dans ses considérations sur l'historicité, Husserl est d'emblée animé par une visée généralisante - celle de dégager, sur l'exemple fourni par la géométrie, l'eidos de l'histoire des sciences, puis de toute pratique culturelle, puis de l'histoire universelle - qu'il n'a finalement ressaisi, dans l'histoire de la géométrie, que des structures très générales, voire purement formelles, a priori susceptibles de convenir ou d'être transposées à tout niveau d'historicité en général?

\section{Structures eidétiques de l'historicité de la géométrie}

De fait, quelles sont les structures de l'historicité que Husserl parvient à dégager depuis le paradigme de l'histoire de la géométrie ?

En premier lieu, le thème essentiel de «L'origine de la géométrie » réside dans le problème de la constitution transcendantale de l'origine ou du sens originel de la géométrie, c'est-à-dire de son instauration primitive ou de son institution originelle. Une telle enquête est sous-tendue par une thèse : celle selon laquelle la genèse de la géométrie est redevable à des actes proto-instaurateurs (urstiftend) qui en ont fixé l'orientation théorétique et thématique, et ainsi déterminé

\footnotetext{
${ }^{4}$ E. Husserl, «Der Ursprung der Geometrie », Hua VI, p. 365 (trad. J. Derrida, L'origine de la géométrie, Paris, PUF, 1962, p. 174).
} 
l'horizon de tâches épistémiques. L'historicité de la géométrie renvoie par là à une "acquisition primitive" (erstes Erwerben), à des "activités créatrices » (schöpferische Aktivitäten) ou une " effectuation productrice » (Leisten) qui en ont établi le sens à l'origine ${ }^{5}$.

En second lieu, ce sens s'est maintenu à l'identique à travers l'histoire, grâce au procès de traditionalisation intergénérationnelle de la géométrie : la géométrie " ne cesse jamais d'avoir cours avec ce sens même » qui a été fixé à l'origine, et qui ouvre un « horizon d'avenir géométrique de même style ${ }^{6}$; le sens de la géométrie possède ainsi une "objectivité "idéale" » ("ideale" Objektivität), c'est-à-dire une identité omnisubjective (possédant une validité pour quiconque, pour tout sujet en général) et supra- ou omnitemporelle (überzeitlich: pour tout temps ou toute époque possible) qui se transfère à toutes ses configurations spécifiques (théorèmes, énoncés, objets) ${ }^{7}$. Et s'il en est ainsi, c'est parce qu'une telle identité de sens fait constamment l'objet d'une réactivation ou réinstauration (Wiederstiftung, Nachstiftung) qui opère la synthèse continuelle (kontinuierliche Synthesis) des nouveaux acquis de la discipline avec les anciens; l'identité omnitemporelle de la discipline se réfère donc à une intersubjectivité transcendantale ouverte, une "chaîne ouverte de générations de chercheurs connus ou inconnus $»^{8}$.

En troisième lieu, l'objectivité idéale de la géométrie, sa constance pour tout sujet et en tout temps (à savoir celle des axiomes et des théorèmes, mais aussi celle des objets sur lesquels ils portent et des états de chose qu'ils visent) a pour condition de possibilité son incarnation linguistique dans un système de signes, de notations elles-mêmes idéales, indéfiniment répétables comme les mêmes dans une multiplicité ouverte d'occurrences : l'objectivité idéale de la discipline théorétique présuppose à titre de condition celle de la langue - sans pour autant $\mathrm{s}^{\prime} \mathrm{y}$ réduire, dans la mesure où l'idéalité des signifiants ne prend sens que par celle des objectités qu'ils désignent ${ }^{9}$. Par là, elle se réfère derechef à une intersubjectivité transcendantale limitée, à savoir un « horizon de nous » (Wir-Horizont) ou une «communauté de langage immédiate et médiate» (unmittelbare und mittelbare Sprachgemeinschaft). Dans la mesure, cependant, où l'objectivité idéale de la géométrie n'est pas liée (gebunden) à un système de signes particulier, mais demeure "identiquement la même dans la "langue originale"

\footnotetext{
${ }^{5}$ Ibid., p. 367 (trad. p. 177 et p. 178).

${ }^{6} \mathrm{Ibid}$., p. 365 et 367 (trad. p. 173 et p. 177).

${ }^{7}$ Ibid., p. 368 (trad. p. 179).

${ }^{8}$ Ibid., p. 367 (trad. p. 177).

${ }_{9}^{9}$ Ibid., p. 368 (trad. p. 180).
} 
d'Euclide et dans toutes les "traductions" » et " est encore une fois la même en toute langue $»^{10}$, elle est de nature translinguistique et implique la possibilité idéale d'un système d'équivalences entre toutes les langues qui, par des expressions différentes, dénotent toutes les mêmes objectités; de ce fait, la géométrie ne se réfère plus simplement à l'intersubjectivité effective des chercheurs vivants, mais à l'horizon indéfiniment extensible d'une «co-humanité », voire de $l^{\prime}$ « humanité en général », «infinité ouverte ${ }^{11}$. Toutefois, une telle idéalité omnisubjective et translinguistique demeure fragile : un système de notations du passé peut devenir totalement opaque aux générations futures, de sorte qu'en dépit de l'idéalité linguistique, le sens originel de la géométrie est toujours susceptible d'une sédimentation recouvrante, ainsi que d'une réactivation découvrante qui le remet au jour ${ }^{12}$.

Enfin, des actes de théorisation intramathématique, voire de la position (même primitive) des seuls axiomes, on est renvoyé à une "formation de sens plus primitive» (primitivere Sinnbildung) nécessaire à titre de «niveau préalable » $(\text { Vorstufe })^{13}$. En effet, les axiomes et théorèmes ne créent pas les objectités sur lesquelles ils portent, mais énoncent quelque chose à propos de telles objectités, qui doivent être préalablement données avant toute énonciation de leurs propriétés ; aussi l'évidence des axiomes se réfère-t-elle en retour à une "évidence originaire» (ursprüngliche Evidenz), qui est donatrice «des idéalités originaires à partir des données préscientifiques du monde de la culture ${ }^{14}$; en effet, les idéalités exactes point, droite, cercle, plan, etc., doivent avoir été produites à partir des idéalités morphologiques, descriptives et anexactes du ponctuel, du droit, du rond, du plat, etc., qui renvoient à la fois à la typification du champ perceptif en formes frappantes et à des formes de praxis artisanale (menuiserie, arpentage, etc.).

Quels enseignements essentiels tirer de ces analyses? Nous en dégagerons deux.

Tout d'abord, l'accent est mis sur l'invariance omnitemporelle $d u$ sens de la géométrie depuis son instauration primitive : quels que puissent être les enrichissements et modifications apportés au cours de l'histoire au champ des idéalités géométriques, la géométrie considérée comme discipline "ne cesse jamais d'avoir cours avec ce sens même » et

\footnotetext{
${ }^{10}$ Idem (trad. p. 179-180).

${ }^{11}$ Ibid., p. 369 (trad. p. 182).

12 Ibid., p. 371-372 (trad. p. 186-187).

${ }^{13}$ Ibid., p. 367 (trad. p. 178).

14 Ibid., p. 375 (trad. p. 194).
} 
« demeure à travers toutes ses formes "la" géométrie »15. L'identité omnitemporelle de la géométrie est garantie par le maintien à l'identique, à travers le procès historique de traditionalisation et de synthèse continuelle des acquis, du sens institué à son origine. L'archè se confond ainsi avec le telos : l'origine possède un sens téléologique, au sens où elle ouvre à la géométrie l'horizon programmatique de ses tâches : être la science de l'espace, de toutes les figures spatiales, et les soumettre à une mesure exacte - géo-métrie. L'horizon historique possède par là sa "structure eidétique » ${ }^{16}$, à savoir une structure de vectorisation téléologique qui renvoie à une instauration originelle du sens; se dévoile ainsi l' «a priori structurel» de l'historicité, comme unité de l'instauration primitive du sens, de sa permanence omnitemporelle (idéale) dans le processus de traditionalisation et de sa vectorisation téléologique, qui fait de toute son histoire un procès orienté de remplissement progressif de la visée initiale de sens - à savoir déterminer l'espace et ses formes. Cette position implique la thèse $d u$ continuisme historique : il y a homogénéité génétique de l'histoire, dans la mesure où elle se réduit à un enrichissement progressif du champ des idéalités, que ne marque aucune rupture et dont le terme ne possède pas un sens distinct vis-à-vis de l'orée; la seule différence entre l'origine et la fin réside dans le degré de remplissement.

Ensuite, Husserl maintient la thèse de l'idéalisme transcendantal ou constitutif, dans une formulation il est vrai quelque peu anthropologisée : "tout traditional est né d'une production humaine » (aus menschlicher Leistung geboren $)^{17}$, et de manière générale tout fait de culture « est une configuration issue d'un acte humain de formation » (Gebilde aus einem menschlichen Bilden) ${ }^{18}$. S'applique ainsi, aux faits et disciplines culturels, la thèse de l'idéalisme constitutif : le sens originel, idéal et omnitemporel de ces disciplines est engendré par un acte subjectif d'instauration primitive, qui ouvre un horizon de reprise, d'enrichissement et de réélaboration du sens par une communauté intergénérationnelle de co-sujets œuvrants. L'unité noématique de l'histoire de la géométrie se révèle simple corrélat intentionnel de la subjectivité proto-instauratrice et de l'intersubjectivité ouverte des co-géomètres, du nous théoricien qui pose à nouveau le sens initial pour l'enrichir. C'est la raison pour laquelle Foucault discernait, entre

\footnotetext{
${ }^{15}$ Ibid., p. 365 (trad. p. 173).

${ }^{16}$ Ibid., p. 378 (trad. p. 199).

${ }_{17}$ Ibid., p. 366 (trad. p. 176).

18 Ibid., p. 379 (trad. p. 202).
} 
ces deux thèses de continuité historique et de constitution par la conscience, une connexion nécessaire.

De là s'ensuit une double question.

D'une part, cette structure eidétique homogène et continue de l'histoire de la géométrie, à la fois archéologique et téléologique, et fondée sur la permanence idéale d'un même sens, est-elle une véritable donnée phénoménologique ? Y a-t-il véritablement continuité de l'histoire de la rationalité mathématique, ou bien existe-t-il au contraire des ruptures épistémologiques entre modalités hétérogènes de la rationalité mathématicienne, entre des espaces de pensée distincts?

D'autre part, l'unité de l'histoire est-elle vraiment garantie par l'identité d'une instance transcendantale qui serait à la fois initialement productrice, puis reproductrice et réélaboratrice? Si l'histoire de la rationalité devait s'avérer scandée par des fractures et se scinder en des époques distinctes marquées par des types de mathesis foncièrement hétérogènes, quelle serait alors la logique qui rend compte du passage d'une mathesis à une autre ? Est-ce bien le sujet transcendantal qui est l'instaurateur des ruptures de style, des mutations de la rationalité mathématique ? Et ce sujet pur peut-il être autre chose que le produit de la purification par laquelle on transforme en sujet anonyme un mathématicien singulier et inventif (par exemple Dedekind, Frege ou Cantor) ? Ou bien la transition d'une mathesis à une autre relève-t-elle d'une dimension autre que celle du sujet transcendantal et de ses actes constituants? Une dimension qui serait asubjective, et semblable à quelque espace de déploiement et de transformation des configurations du savoir?

\section{Phénoménologie de l'engendrement de nouvelles idéalités}

Une phénoménologie de l'historicité des idéalités mathématiques ne saurait cependant se contenter de thématiser les actes d'instauration originaire (Urstiftung), de réinstauration (Wieder- oder Nachstiftung) et de réactivation (Reaktivierung). L'objet essentiel d'une théorie de l'historicité des mathématiques, ce sont plutôt les modalités de $l^{\prime}$ «élaboration continuée » (Fortarbeit) ou "synthèse continuelle » (kontinuierliche Synthesis) ${ }^{19}$ qui engendre ou découvre de nouvelles idéalités (propriétés d'objets ou d'ensembles d'objets, théories mises sous forme déductive, champs d'objets). Comment caractériser une telle synthèse productrice ou constituante autrement que par les seuls

${ }^{19}$ Ibid., p. 366-367 (trad. p. 175 et p. 177). 
concepts de réinstauration et de réactivation d'un sens ou d'un projet originel - lesquels ne sauraient rendre compte du caractère polymorphe de l'activité de théorisation mathématicienne, mais seulement éclairer dans une généralité vague les idées génériques d'arithmétique, de géométrie, d'Analyse, etc. ?

$\mathrm{Au}$ sein de la doctrine husserlienne, ce n'est pas dans «L'origine de la géométrie » que l'on peut trouver l'élucidation de la ou des structures de l'émergence du nouveau en mathématiques - mais ailleurs, dans la conceptualité propre à la phénoménologie transcendantale classique et dans les considérations de la Krisis.

Tout d'abord, les Ideen I livrent une explicitation du type d'acte qui constitue des idéalités à partir d'objets donnés, des objets d'ordre supérieur à partir d'objets d'ordre inférieur ${ }^{20}$. $\mathrm{Si}$, comme le reconnaît ultérieurement Husserl dans les Cartesianische Meditationen, la synthèse est la forme originaire (Urform) de toute conscience d'objet ${ }^{21}$, il faut cependant faire la distinction entre deux formes fondamentales de synthèse: d'une part les synthèses continues qui, par exemple dans la perception spatiale, lient ensemble les diverses facettes perspectives pour leur conférer le sens de modes de présentation d'un seul et même objet; de l'autre, les synthèses articulées (gegliedert), syntaxiques ou polythétiques, qui engendrent un objet d'ordre supérieur à partir de positions préalables d'objets d'ordre inférieur ${ }^{22}$. Or, c'est de ce dernier type que sont les synthèses de la pensée mathématique: ce sont des modalités d'unification d'actes discrets en l'unité d'une synthèse articulée, c'est-à-dire en un "acte d'ordre supérieur» (Akt höherer Ordnung); des actes de mise en forme syntaxique d'une multiplicité d'objets, puis de conversion des actes polythétiques en un acte monothétique producteur d'un nouvel objet. L'exemple donné par Husserl est celui de la formation d'un ensemble à partir de la conjonction, rapportée à une pluralité d'objets donnés : on peut attribuer une même propriété à plusieurs objets de manière à former une conjonction de propositions $($ A est $p) \wedge(\text { B est } p)^{\wedge}(\mathrm{C} \text { est } p)^{\wedge} \ldots$, puis former un sujet pluriel par réunion des objets ainsi concernés par ces prédications : (A et $\mathrm{B}$ et $\mathrm{C}$...) sont $p$ s; par conversion de cette réunion en un acte monothétique ou par nominalisation de la forme syntaxique de la conjonction, on forme alors un nouvel objet, l'ensemble contenant comme éléments les objets $A, B, C$, etc. : $\{A, B$,

\footnotetext{
${ }^{20}$ E. Husserl, Ideen zu einer reinen Phänomenologie, Bd I, §118-119, Hua III/1, p. 272-277 (trad. P. Ricœur, Idées directrices pour une phénoménologie pure, Paris, Gallimard, 1950, p. 402-407).

${ }^{21}$ E. Husserl, Cartesianische Meditationen, § 17, Hua I, p. 77 (trad. M. de Launay éd., Méditations cartésiennes, Paris, PUF, 1994, p. 84).

22 E. Husserl, Ideen I, § 118, Hua III/1, p. 273-274 (trad. p. 403-404).
} 
$\mathrm{C}, \ldots\}^{23}$. Il y a là un acte d'engendrement originaire (ursprüngliches Erzeugen ${ }^{24}$ qui, d'un champ d'objets préconstitués, permet de passer à un domaine d'objets supérieurs par une simple opération syntaxique-formelle sur ces objets, puis par conversion nominalisante de cette opération syntaxique. La syntaxe y possède une double fonction: c'est la forme de l'opération effectuée sur les objets d'ordre inférieur, mais aussi, conjointement, la structure intrinsèque qui caractérise les nouvelles objectités engendrées ${ }^{25}$.

On peut donc donner une première réponse à la question de savoir quelle est la nature de la "synthèse continuelle » qui opère au sein d'une discipline et en tisse l'histoire : il s'agit d'une synthèse articulée ou syntaxique, qui engendre des objectités idéales d'ordre toujours croissant. La structure de l'historicité qui est corrélative à cet étagement de synthèses articulées obéit à un modèle architectonique et continuiste: depuis les archi-objets que sont les étants donnés par la perception sensible, on engendre successivement, couche par couche et sans lacune dans la concaténation des strates, de nouvelles idéalités ; il s'agit d'une édification et d'un enrichissement progressifs du domaine des objets idéaux. Une telle structure est régie par la relation fondamentale de fondation (Fundierung), mise en évidence par Husserl dès les Recherches logiques: un objet d'ordre supérieur est fondé sur un ensemble d'objets d'ordre inférieur, dans la mesure où sa constitution présuppose ces derniers comme prédonnés.

Tel n'est cependant pas le modèle exclusif d'historicité pensé par Husserl ; il en présente en effet, au § 9f de la Krisis, un modèle à la fois téléologique et discontinuiste. Husserl s'attache de fait à mettre en évidence l'orientation téléologique de l'histoire des mathématiques comme une tendance vers une "formalisation tout à fait universelle » (völlig universale Formalisierung) ${ }^{26}$, dont les paliers de réalisation essentiels sont les suivants. Premièrement, un mouvement d'algébrisation de la pensée qui commence avec l'Algèbre de Viète, pour se prolonger avec Descartes et Leibniz: il s'agit de l'élaboration du mode de notation littéral ou algébrique, qui permet le développement d'une pensée des nombres et des grandeurs affranchie de toute référence à des nombres et grandeurs particuliers - un tel développement pas-

\footnotetext{
${ }^{23}$ Ibid., § 119, p. 275-276 (trad. p. 405-407). Cf. Erfahrung und Urteil, § 61, p. 292-296 (trad. p. 295299).

${ }^{24}$ E. Husserl, Formale und transzendentale Logik, §63, Hua XVII, p. 175-176 (trad. S. Bachelard, Logique formelle et logique transcendantale, Paris, PUF, 1957, p. 226-228).

25 Ibid., § 42d, Hua XVII, p. 119-120 (trad. p. 155-156). Cartesianische Meditationen, § 18, Hua I, p. 80 (trad. p. 88).

${ }_{26}$ E. Husserl, Krisis, § 9f, Hua VI, p. 44 (trad. p. 52).
} 
sant par l'élaboration cartésienne de la géométrie analytique et le développement de la théorie des fonctions, avant de culminer avec l'arithmétisation de l'Analyse par Cantor et Dedekind. Deuxièmement, un mouvement d'axiomatisation des théories, que Husserl pense comme une analytique formelle, c'est-à-dire une théorie des formes de théorie déductive libre de toute référence précise (donc énonçant des formes d'axiomes à propos d'objets indéterminés, sinon par les relations posées dans ces axiomes) et des formes de champ d'objets associées aux premières (auquel il donne le nom de multiplicité définie); il s'agit là d'une formalisation universelle des théories et des domaines d'objets, dans la mesure où les unes comme les autres sont déliées de toute référence à une teneur réale, voire de toute référence déterminée - dans l'esprit de Husserl, l'Idée d'une telle théorie universelle des formes de théorie et de champ associé avait été vue par Leibniz, sous le titre de mathesis universalis ${ }^{27}$. Le troisième palier du procès historique de formalisation est l'élaboration purement analytique, par Riemann, des multiplicités $n$-dimensionnelles, c'est-à-dire de formes catégoriales (analytiques) d'espace affranchies de toute référence proprement géométrique et pourvues de courbure quelconque, dont l'espace tridimensionnel euclidien n'est qu'un cas particulier ${ }^{28}$.

Quelles conclusions tirer de telles considérations téléologiques?

La conséquence essentielle est la suivante : contrairement au premier modèle, continuiste, de l'enrichissement synthétique progressif du champ des idéalités ou de l'édification stratifiée d'objets d'ordre supérieur, se fait jour un modèle discontinuiste de l'histoire, strié par des ruptures épistémologiques. Ainsi, au $\S 8$, Husserl insistait-il sur la «violente mutation de sens" (gewaltige Sinnwandlung) qui, des mathématiques grecques à la mathématique contemporaine, vient affecter à la fois le sens des mathématiques et leur fonction pour la science physique ${ }^{29}$. Chez les Grecs, en effet, régnait une dualité des mathématiques, lesquelles se scindent en géométrie (science rapportée à l'espace intuitif, dont les figures sont soumises à un acte

\footnotetext{
${ }^{27}$ Ibid., p. $44-45$ (trad. p.52-53). Les recherches leibniziennes ont révélé à quel point cette interprétation husserlienne de la mathesis universalis leibnizienne était pour une large part fantasmé- cf. D. Rabouin, "Husserl et le projet leibnizien d'une mathesis universalis », Philosophie, $\mathrm{n}^{\circ}$ 92, 2006, p. 13-28. Bolzano nous semble avoir été une médiation essentielle dans l'élaboration de cette interprétation, notamment le texte de 1810 intitulé Allgemeine Mathesis, Gesamtausgabe II A 5, p. 13-64 (trad. coord. C. Maigné et J. Sébestik, « Mathesis universelle » in B. Bolzano, Premiers écrits. Philosophie, logique, mathématique, Paris, Vrin, 2010, p. 138-180).

${ }^{28}$ E. Husserl, Krisis, §9f, Hua VI, p. 45 (trad. p. 53). Formale und transzendentale Logik, §30, Hua XVII, p. 97-98 (trad. p. 127-129).

${ }^{29}$ E. Husserl, Krisis, § 8, Hua VI, p. 18 (trad. p. 25).
} 
d'idéalisation exactifiante) et la théorie des nombres et des grandeurs (élaborée dans une abstraction formalisante qui évacue toute teneur réale des objets). À l'époque moderne, en revanche, ont conjointement lieu l'unification de la mathématique (envisagée comme une mathesis purement formelle, théorie des champs d'objets indéterminés susceptibles de satisfaire un système déductif de formes propositionnelles) et le passage d'un a priori fini à un a priori infini - celui de la construction axiomatique des formes de théorie et des domaines d'objets associés, où toutes les propositions seraient décidables, donc tous les prédicats d'objets arrêtés par les axiomes ou les théorèmes : c'est en celle-ci que réside la "nouveauté inouïe» (das unerhört Neues), à savoir la transition d'une mathématique finie, parce que référée à des champs d'objets spécifiés (espace plan et ses figures, espace tridimensionnel, nombres, grandeurs...) à une mathématique infinie, baptisée ainsi parce qu'elle thématise désormais des domaines d'objets quelconques dominables par une théorie déductive. Cette inflexion essentielle de l'histoire des mathématiques vaut comme exemple et indice d'une thèse générale sur leur type d'historicité : il existe des coupures épistémologiques qui opèrent une mutation de style de la rationalité mathématique et séparent des espaces de pensée hétérogènes.

De là s'ensuit une question fondamentale : peut-on élucider phénoménologiquement la structure du passage d'un mode de pensée à un autre, et rendre compte de l'instance productrice qui opère de telles mutations?

Pour Husserl, la réponse est donnée avant même que la question ne soit posée. Dans l'histoire a en effet lieu le déploiement d'une téléologie interne, d'une dynamis intrinsèque qui, au-delà de la diversité voire de la disparité des théories mathématiques, aimante de façon unitaire le devenir général de la raison mathématicienne vers un pôle téléologique : à savoir la tendance vers une «formalisation tout à fait universelle» (völlig universale Formalisierung) ${ }^{30}$ - formalisation toujours plus poussée, que dans les Recherches logiques Husserl avait évaluée positivement, comme un affranchissement des possibilités de la pensée catégoriale vis-à-vis de toute adhérence à la réalité mondaine $^{31}$, avant de lui conférer, dans la Krisis et "L'origine de la géométrie », le sens négatif d'un "évidement du sens » (Sinnentleerung) de la pensée géométrique joint à la «technicisation » (Technisierung),

\footnotetext{
${ }^{30}$ Cf. supra.

${ }^{31}$ E. Husserl, Logische Untersuchungen, Prolegomena zur reinen Logik, § 70, Hua XVIII, p. 252-253 (trad. Recherches logiques, tome I, Paris, PUF, 19692, p. 276-277).
} 
c'est-à-dire la tendance vers une mathématique formaliste des règles du jeu ${ }^{32}$.

Quant à la nature de l'instance productrice des fractures épistémologiques, il ne fait aucun doute pour Husserl qu'il s'agit de la subjectivité constituante. De même que c'est le sujet transcendantal qui constitue toutes les idéalités mathématiques, c'est également lui qui instaure et fixe les Idées téléologiques nouvelles qui ouvrent un nouvel horizon de tâches mathématiques; en particulier, c'est lui qui instaure la tâche programmatique indéfinie de formalisation croissante des mathématiques. $\mathrm{Si}$, en effet, la thèse ontologique fondamentale de Husserl consiste dans la position de l'absoluité de la conscience pure et dans la reconnaissance du fait que tout étant autre que cette dernière se réduit à une configuration de sens constituée (c'est-à-dire visée et validée) en elle et par elle ${ }^{33}$, une telle thèse ne souffre aucune exception, et vaut pour les Idées téléologiques qui vectorisent l'histoire comme pour n'importe quel étant: de même qu'en général tout sens renvoie à une donation de sens (Sinngebung) ${ }^{34}$, tout sens historique renvoie à une proto-instauration (Urstiftung) qui le fixe pour la première fois, et ainsi toute Idée programmatique nouvelle doit avoir été fixée par une telle Urstiftung. Jamais Husserl ne destitue le sujet transcendantal de sa fonction originairement productrice ou de son statut de fondement de tout sens, pour lui substituer une dynamis asubjective et anonyme qui appartiendrait, de façon immanente, aux champs d'idéalités eux-mêmes.

Un parallèle entre l'histoire de la philosophie et celle des mathématiques suffira à le faire saisir. Pour Husserl, l'histoire de la philosophie tout entière est un progrès téléologique de réalisation de la forme finale qu'est la phénoménologie transcendantale, et ce remplissement s'accomplit depuis la protofondation platonicienne de l'Idée de philosophie comme science, puis par les trois réinstaurations partielles que sont les philosophies cartésienne, humienne et kantienne. Or, cette dynamis est-elle une propension objective et anonyme qui, sans l'efficace d'aucun acte instaurateur d'un sujet, animerait de l'intérieur l'histoire et la pousserait vers la réalisation de l'Idée de phénoménologie constitutive ? Non ! Cette Idée a été constituée par Husserl lui-même (comme instance de la conscience pure) puis par tout autre philosophe qui s'approprie ses thèses, et c'est depuis cette Idée constituée qu'une méditation historique en retour

\footnotetext{
${ }^{32}$ E. Husserl, Krisis, § 9d, Hua VI, p. 45 (trad. p. 53).

${ }^{33}$ E. Husserl, Cartesianische Meditationen, $\S 41$, Hua I, p. 117-118 (trad. p. 132-134).

${ }^{34}$ E. Husserl, Ideen I, § 55, Hua III/1, p. 120 (trad. p. 183).
} 
(historische Rückbesinnung) permet a parte post d'interpréter les philosophies cartésienne, humienne et kantienne comme en étant des préfigurations intentionnelles partielles. Or, il en va de même pour l'histoire des mathématiques: la tendance vers une formalisation toujours plus poussée n'habite pas de l'intérieur cette histoire comme une dynamis objective, anonyme et non constituée ; c'est une tendance qui a été instituée dans sa pureté par les mathématiciens contemporains (Hilbert et Riemann), et c'est depuis cet acte contemporain de constitution que, par un geste épistémologique rétrospectif, on peut éclairer en retour les créations de Viète, Nicolas Oresme, Descartes et Leibniz comme participant de ce mouvement de formalisation. Si Husserl parle de «force pulsionnelle » (Triebkraft) habitant de l'intérieur le devenir historique pour le vectoriser ${ }^{35}$, il ne s'agit cependant pas d'une pulsion aveugle et non constituée; dynamis et telos n'ont au contraire de sens que depuis leur constitution contemporaine, et grâce à l'interprétation rétrospective qu'impose cette dernière.

En conclusion, les Idées qui ouvrent un nouvel horizon de recherche et instituent une rupture épistémologique ne sont pas une exception à la thèse de l'idéalisme transcendantal : comme tout étant en général, elles sont constituées par des actes noétiques du sujet pur.

\section{Modalités de mutation historique du champ des idéalités : une « histoire qui n'est pas une histoire »}

Est-il possible de décrire phénoménologiquement, dans leurs traits eidétiques, les modalités typiques d'émergence de nouveaux champs d'idéalités, voire de nouvelles formes de rationalité mathématique?

La solution husserlienne nous laisse en effet doublement insatisfaits.

D'une part, le recours à un acte instaurateur du sujet pur n'explique rien : certes, toute configuration noématique de sens renvoie à des actes noétiques qui l'instaurent; mais si, au-delà de cette structure de renvoi formelle à la corrélation noético-noématique, on veut véritablement rendre compte de telle ou telle inflexion précise de l'histoire des mathématiques (par exemple, l'arithmétisation de l'Analyse par Dedekind), il nous faut alors prendre en considération les champs d'objets idéaux déjà constitués à l'époque antérieure, afin d'en dégager la structure de manque et de ressaisir la tension entre certaines exigences opératoires (ou thématiques) et la nature du

\footnotetext{
${ }^{35}$ E. Husserl, Krisis, $§ 5$ et 15, Hua VI, p. 10 et p. 72 (trad. p. 17 et p. 82).
} 
domaine d'objets disponible - bref, spécifier la motivation qui a présidé à l'acte d'instauration de nouvelles idéalités. Or, une telle motivation doit en dernière instance résider dans quelque structure de manque propre au champ d'objets lui-même, c'est-à-dire dans des traits du champ noématique.

D'autre part, la prétention de l'élucidation phénoménologique est eidétique : il ne s'agit pas seulement d'expliciter les motivations de telle rupture précise au sein de l'histoire des mathématiques, mais de ressaisir les structures eidétiques de telles ruptures en général, c'està-dire la légalité de l'engendrement de nouvelles idéalités et de la mutation du mode de rationalité. Or, le modèle téléologique husserlien le permet-il ? N'oublions pas l'une de ses caractéristiques essentielles: le telos assigné à l'histoire n'est pas un pôle en soi qui assignerait à la Geschichtlichkeit (l'histoire telle qu'elle a lieu) une propension objective vers un but, mais se confond avec l'Idée d'une forme finale (de la philosophie, ou des mathématiques) qui a de facto été instaurée à l'époque contemporaine ${ }^{36}$; en $\mathrm{d}^{\prime}$ autres termes, la dynamis historique $\mathrm{n}^{\prime}$ est que le corrélat d'une prise de conscience rétrospective, par une discipline prise à son état ultime (la phénoménologie transcendantale, et la mathématique formalisée), de l'historicité qui a permis son apparition et son développement. C'est dire que, lorsque changera la forme finale qui sert de terminus a quo à cette interprétation rétrospective, changeront également les paliers historiques marquants qui en ont scandé l'histoire!

De fait, tel semble bien être le problème posé par toute tentative d'idéation cherchant à élucider l'essence de l'activité mathématicienne ou de ses moments de rupture : l'histoire des mathématiques étant, dans les termes husserliens, une "synthèse continuelle ", c'està-dire une production incessante de nouvelles théories, de nouveaux champs d'objets et de nouveaux procédés démonstratifs, il s'avère impossible de clore sub specie aeternitatis la liste des types d'actes constituants de la pensée mathématique. Ainsi Cavaillès évoque-t-il, dans «La pensée mathématique », l'impossibilité de "recenser, d'une façon définitive et exhaustive, les procédés que [le mathématicien] emploie. On peut [seulement] les recenser à un moment donné ${ }^{37}$. C'est précisément l'historicité des mathématiques, la transformation des domaines d'objets et des procédures, qui semble frapper d'inanité toute volonté d'élucider l'eidos de l'acte constituant les idéalités et, à

\footnotetext{
${ }^{36}$ Ibid., § 14, Hua VI, p. 71 (trad. p. 81).

37 J. Cavaillès, "La pensée mathématique », in CEuvres complètes de philosophie des sciences (désormais noté O. C.), Paris, Hermann, 1994, p. 600.
} 
plus forte raison, de l'acte instituant une mutation de la rationalité mathématique elle-même.

Ce nonobstant, toute visée eidétique n'est pas pour autant abandonnée : si l'on ne saurait ressaisir un eidos invariant et omnitemporel de l'activité mathématicienne, il s'avère en revanche possible de penser l'eidos de l'historicité de la mathématique. En effet, l'histoire des mathématiques a pour trait essentiel que, caractérisée par l'historicité de contenus et de domaines idéaux, elle ne relève pas de l'événementialité contingente de faits singuliers, mais d'une forme de nécessité inhérente aux idéalités elles-mêmes : c'est une « histoire qui n'est pas une histoire $»^{38} ;$ " [1]'histoire des mathématiques semble, de toutes les histoires, la moins liée à ce dont elle est le véhicule [...] il y a une objectivité, fondée mathématiquement, du devenir mathématique ${ }^{39}$; " [i]l n'y a rien de si peu historique [...] que l'histoire mathématique. Mais rien $\mathrm{d}^{\prime}$ aussi peu réductible, dans sa singularité radicale $\gg^{40}$; l'activité mathématique est un « devenir historique original », et il y a une « [d]ialectique de l'histoire mathématique : libération du contingent par l'effectif » ${ }^{41}$; «le progressif est d'essence et les décisions qui le négligent se perdent dans le vide ${ }^{42}$.

Comment, dès lors, caractériser cette progressivité essentielle aux champs idéaux? Comment ressaisir et analyser la nécessité spécifique qui régit le devenir autonome des mathématiques?

$\mathrm{Si}$, lors de la séance de la Société française de philosophie, Cavaillès semble se contenter de ne livrer, de manière empirique et descriptive, que « quelques-uns des procédés employés par les mathématiciens ", relatifs à une situation mathématique déterminée et peutêtre caractérisés par leur adhérence à celle-ci, il ambitionne cependant, en présentant ces opérations, $\mathrm{d}^{\prime}$ " apercevoir des nécessités sous l'enchaînement des notions et des procédés », à savoir la nécessité $\mathrm{d}^{\prime}$ une «dialectique fondamentale des mathématiques " $^{43}$ ou d'un

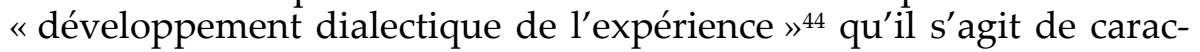
tériser. Pourquoi dialectique? Et à quel type de dialectique est-il fait référence ? La logique de l'élargissement de l'expérience et de l'émergence de nouvelles idéalités correspond au «triple rôle de la géné-

\footnotetext{
38 J. Cavaillès, « Mathématiques et formalisme », in O. C., p. 664.

${ }^{39}$ J. Cavaillès, Philosophie mathématique, p. 27-28 (O. C., p. 225-226).

40 J. Cavaillès, Méthode axiomatique et formalisme, p. 176 (O. C., p. 184).

${ }^{41}$ J. Cavaillès, «Réflexions sur le fondement des mathématiques », in O. C., p. 578.

42 J. Cavaillès, Sur la logique et la théorie de la science, p. 70 (O. C., p. 552).

43 J. Cavaillès, «La pensée mathématique », in O. C., p. 602, p. 600 et p. 601.

${ }^{44}$ J. Cavaillès, Méthode axiomatique et formalisme, p. 177 (O. C., p. 185).
} 
ralisation $\gg^{45}: c^{\prime}$ est toujours une modalité de généralisation qui caractérise la progressivité des mathématiques, leur passage d'une situation à une autre ; à savoir la transition d'un champ d'idéalités à un autre, le premier possédant le statut de singularité relativement au second, qui se caractérise par sa généralité englobante. Pourquoi ? Seule l'analyse de ces trois modalités permettra de le déterminer.

La première est l'idéalisation ou adjonction d'idéaux, dont le modèle est donné par les élargissements successifs de l'ensemble des nombres chez Dedekind ${ }^{46}$ : une opération (par exemple, la soustraction) se voit imposer une limitation extrinsèque par la nature particulière des nombres sur lesquels on l'opère (il est impossible, dans $\mathbb{N}$, de soustraire d'un entier un entier plus grand que lui); il est alors possible $\mathrm{d}$ 'affranchir l'opération de cette limitation extrinsèque en effectuant une adjonction d'éléments idéaux au domaine primitif (ici, en ajoutant les entiers négatifs $)^{47}$. On a ainsi un modèle d'engendrement opératoire de nouvelles idéalités par réduction des restrictions extrinsèques imposées à une opération (ici, la soustraction) par son champ d'application initial : transformation de l'opération primitive en loi de composition interne par extension de l'ensemble primitif des objets (ici, N), qui permet de définir l'opération exclusivement par ses propriétés formelles. Mais ce à une double condition, qui est une double connexion entre le domaine primitif et le domaine élargi : l'une, réductionniste, qui consiste à retraduire dans le premier les objets et opérations du second (un relatif est un couple d'entiers naturels respectant certaines lois opératoires) ; l'autre, condition d'invariance, qui exige la restitution du domaine primitif par élimination des idéaux (on obtient les entiers en éliminant la partie négative des relatifs) $)^{48}$.

La deuxième est la thématisation ${ }^{49}$ : réflexivité opératoire, transformation de l'opératoire en thématique ou de l'opération en objet. On peut en effet transformer une opération, au départ défini sur un champ d'objets déterminé, en objet ou en individu d'un domaine sur lequel on peut répéter cette même opération; ainsi peut-on élaborer la topologie des transformations topologiques, de même que l'on

\footnotetext{
${ }^{45}$ Ibid., p. 172 (O. C., p. 180).

${ }^{46} \mathrm{~J}$. Cavaillès, «Réflexions sur le fondement des mathématiques » et «La pensée mathématique ", in O. C., p. 579 et p. 602, Méthode axiomatique et formalisme, p. 172 (O. C., p. 180).

47 J. Cavaillès, Méthode axiomatique et formalisme, p. 97 (O. C., p. 105).

${ }^{48}$ Idem.

${ }^{49} \mathrm{~J}$. Cavaillès, «Réflexions sur le fondement des mathématiques» et «La pensée mathématique », in O. C., p. 579 et p. 602. Sur la logique et la théorie de la science, p. 30-32 (O. C., p. 512514).
} 
peut itérer la formation de l'ensemble en forgeant la notion d'ensemble de tous les ensembles (itération qui est à l'origine d'antinomies), etc. Une telle modalité peut se penser au sein du seul champ noématique, sans recourir à la notion d'acte subjectif, puisque, pour une même entité idéale, elle relève de la possibilité de passer du statut d'opération à celui d'objet : au départ opération sur un champ d'objets primitif (celui des espaces), une transformation topologique (formation de sous-espace, d'une topologie produit, d'un groupe topologique, homéomorphisme, recollement...) devient objet d'un champ de second ordre, sur lequel on peut à nouveau étudier des transformations topologiques. Aussi Cavaillès traduit-il pour finir ce procédé à partir de la "dualité entre sens posant et sens posé ${ }^{50}$ : le sens posant, c'est le sens opératoire sur le champ primitif; le sens posé, c'est le sens thématique de l'opération devenue objet du nouveau champ ; en s'appliquant au sens posé qu'est l'opération-objet, le sens posant qu'est l'opération redoublée acquiert une signification nouvelle et plus abstraite.

La troisième est baptisée du nom de paradigme: passage de la liaison-acte, effectuée sur des objets déterminés, à la liaison-type effectuée sur des objets quelconques, passage de l'acte à son sens grâce à l'intervention du moment de la variable, du quelconque ou (en termes husserliens) de la formalisation, ou encore passage de la forme d'une matière à une forme pure. Les exemples, nombreux, sont ceux où une relation ou une opération cesse d'adhérer à un domaine particulier pour se définir dans l'abstrait, relativement à des champs d'objets indéterminés : tel est le projet d'une algèbre générale chez Whitehead, définissant des opérations hors de toute relativité à des champs d'objet donnés; tel est encore le cas de l'axiomatisation, qui n'est jamais pure construction dans l'abstrait, mais toujours purification d'une théorie préexistante portant sur des objets naturels (par exemple la géométrie euclidienne dans les Grundlagen der Geometrie de Hilbert, ou l'arithmétique élémentaire dans l'axiomatique de Peano), et qui «dépouille la théorie de tout l'adventice pour la rendre purement elle-même », c'est-à-dire en présenter la structure opératoire et déductive dans toute sa pureté ${ }^{51}$.

Qu'est-ce qui est ici fondamental, et fournit une structure eidétique commune à tous les types de généralisation ici décrits? Il s'avère ici nécessaire de purifier $\mathrm{d}^{\prime}$ une fâcheuse équivoque le terme de généralisation: si ce dernier évoque en général une activité subjective

${ }^{50}$ J. Cavaillès, Sur la logique et la théorie de la science, p. 31 (O. C., p. 513).
${ }^{51}$ J. Cavaillès, Méthode axiomatique et formalisme, p. 88 (O. C., p. 96). 
assurant le passage à un degré plus élevé de généralité, il est impératif, si l'on veut en ressaisir la nécessité intrinsèque, de la dépouiller de son caractère d'acte subjectif. En effet, la nécessité de dépassement, d'autodépassement ou de progressivité appartient aux champs d'idéalités eux-mêmes, et se situe sur le seul plan noématique. Le cas le plus simple est celui de l'élargissement successif des ensembles de nombres : c'est l'impossibilité de pouvoir toujours accomplir la soustraction qui appelle la constitution des entiers négatifs, puis des relatifs; c'est l'écart entre le domaine d'objets primitif et la possibilité opératoire qui requiert un élargissement du premier, c'est-à-dire une bonne extension du domaine d'entités idéales admises comme existantes. En d'autres termes, c'est toujours la "structure des parties révélées " $^{2}$ - à savoir, l'articulation entre un système d'objets et un système d'opérations et de relations - qui impose la position de nouvelles idéalités et la transition d'une situation mathématique à une autre : le champ thématique doit nécessairement présenter une structure lacunaire qui exige une complétion. Et cette complétion prend les trois formes qui ont été dégagées : rabotage des limitations extrinsèques d'une opération liées à son adhérence à un domaine primitif d'application, conversion d'une opération en objet thématique dans un nouveau champ où s'opère une duplication opératoire, enfin passage d'opérations et relations définies sur un champ particulier à leur thématisation abstraite - complétion du champ d'objets, objectivation et répétition de l'opération, passage du particulier au quelconque ou purification de structures adhérentes.

On comprend, dès lors, l'emploi récurrent du concept de dialectique : dialectique signifie ici qu'à un moment donné de l'histoire, le champ des entités intelligibles est porteur de lacunes ou de discrépances entre l'opératoire et le thématique - structure de manque qui exige le passage à un niveau supérieur de thématisation; un équivalent, dans le champ des idéalités, du travail du négatif cher à Hegel. Quelle est alors la place du sujet ? Non que les actes de mathématiciens ne soient nécessaires à la position de nouveaux objets : sans mathématiciens existants, il n'y aurait certes pas d'historicité des mathématiques, pas de production du nouveau. Mais ce n'est pas dans les actes que l'on trouvera la nécessité génératrice qui fait passer du niveau inférieur au supérieur : celle-ci réside dans la structure du champ des idéalités, à savoir dans la manière dont les possibilités opératoires dessinent par avance la nécessité de possibilités thématiques, la position

52 J. Cavaillès, Philosophie mathématique, p. 28 (O. C., p. 226). 
de nouveaux objets, concepts et opérations. «La nécessité génératrice n'est pas celle d'une activité, mais d'une dialectique $»^{53}$.

\section{Première loi de l'historicité : le fonctionnement paradigmatique de la manifestation}

Cet élargissement ne pourrait-il cependant continuer d'être pensé et formulé dans la conceptualité de la phénoménologie husserlienne, au sein d'une noétique générale, comme un acte constituant motivé par les limitations opératoires du champ antérieur? Les concepts complémentaires de constitution (proto-instauration ou proto-engendrement) et de motivation ne peuvent-ils rendre compte de l'émergence de nouvelles idéalités, sans qu'il soit nécessaire d'adopter une thèse d'asubjectivité du procès historique ? Tel est, de fait, l'argument que Derrida opposait à la critique de Husserl par Cavaillès, et à sa substitution d'une dialectique asubjective à l'activité génératrice de la conscience pure $^{54}$ : celle-ci est en effet intrinsèquement dialectique, dans la mesure où elle a toujours pour pendant la passivité du domaine mathématique déjà constitué, passivité sédimentée dans une naïveté objectiviste dont la relativité à la conscience ne se réveillerait que lorsque celle-ci produit un sens de degré supérieur; ainsi l'ensemble des entiers naturels demeure-t-il un domaine inerte, jusqu'à ce qu'il serve de fondement et de motivation à la constitution de nombres de degré supérieur (nombres relatifs, etc.), auquel cas sa relation à la conscience se réactive. Cavaillès n'aurait donc fait qu'absolutiser et rendre asubjectif la dialectique entre sédimentation passive et constitution active qui caractérise l'historicité de la vie constituante.

La référence à une telle activité créatrice de la subjectivité en mathématiques est prégnante depuis les célèbres formules de Dedekind dans la première préface à Was sind und was sollen die Zahlen? : "Les nombres sont de libres créations de l'esprit humain, ils servent de moyen pour saisir plus aisément et plus précisément la diversité des choses $»^{55}$. La formule a souvent été entendue dans le sillage du mé-

\footnotetext{
${ }^{53}$ J. Cavaillès, Sur la logique et la théorie de la science, p. 78 (O. C., p. 560).

54 J. Derrida, «Introduction » à E. Husserl, L'origine de la géométrie, op. cit., p. 157-159.

${ }_{55}$ R. Dedekind, Was sind und was sollen die Zahlen?, in Gesammelte Mathematische Schriften, III, Braunschweig, Vieweg, 1930-1932 (désormais note GMS III); trad. H. Benis-Sinaceur, Que sont et à quoi servent les nombres, in R. Dedekind, La création des nombres, Paris, Vrin, 2008, p. 134). De même : « [...] les progrès les plus grands et les plus féconds en mathématiques et dans d'autres sciences sont dus avant tout à la création et à l'introduction de nouveaux concepts, que rendent
} 
moire de Cauchy sur les imaginaires : l'engendrement de nombres imaginaires consiste à écrire une «formule symbolique » dépourvue de sens dans le domaine initial (par exemple $\sqrt{2}$ dans $\mathbb{Q}$ ou $\sqrt{ }-1$ dans $\mathbb{R}$ ), puis à énoncer pour les formules ainsi introduites des règles de calcul compatibles avec les règles sur les nombres ordinaires, de façon à obtenir un corps d'entités plus large par adjonction extensionnelle ${ }^{56}$. Entendue en ce sens, elle a été critiquée aussi bien par Husserl que par Russell : il est illégitime de postuler l'existence de nouvelles entités par une définition créatrice (par exemple, d'ajouter des nombres irrationnels aux nombres rationnels), car poser une notation absurde revient à créer un "concept absurde ", interdit par l'ancien concept de nombre ; il faut donc abandonner ce dernier pour redéfinir intensionnellement un nouveau concept de nombre (ici, définir les nombres réels : soit comme la limite d'une suite de rationnels comme Cantor, soit comme un segment de rationnels comme Russell) ${ }^{57}$. Ces critiques ne modifient cependant en rien la position fondamentale: même défini de manière intensionnelle et non obtenu par adjonction extensionnelle, le nouveau concept de nombre est toujours engendré par un acte de la conscience, et ce sens doit être validé par des preuves de non-contradiction.

En revenant à Dedekind pour en rectifier l'interprétation, nous allons pouvoir dégager un principe de la productivité mathématique, donc de son historicité.

Il est en effet d'abord nécessaire de rectifier cette interprétation de la formule citée sur les «libres créations de l'esprit humain », entendus comme actes de production par des définitions créatrices ${ }^{58}$. Car Dedekind évoque aussitôt après la «construction purement logique de la science des nombres et [le] domaine continu des nombres

nécessaires le fréquent retour de phénomènes complexes difficilement maîtrisables par les anciens concepts » et "Comment, ensuite, élargir graduellement le concept de nombre, créer le zéro, les nombres négatifs, fractionnaires, irrationnels et complexes, en les ramenant toujours aux concepts antérieurs et sans interférence de représentations de nature étrangère [...] » (ibid., p. 139 et p. 138, nous soulignons).

56 A. L. Cauchy, «Analyse algébrique. Mémoire sur une nouvelle théorie des imaginaires et sur les racines symboliques des équations et des équivalences ", 1847, rééd. in CEuvres complètes de A. L. Cauchy, 1ère série, X, Paris, Gauthier-Villars, 1897, p. 312. Cf. l'analyse de V.Gérard, «Husserl élève de Kronecker et Weierstrass : théorie de la signification, théorie des nombres et théorie des fonctions » in Husserl, J. Benoist (éd.), Paris, Cerf, 2008, p. 17 sqq.

${ }^{57}$ E. Husserl, Manunscrit K I 26, « Husserls Manuskripte zu seinem Göttinger Doppelvortrag von 1901 », Husserl Studies, n²17, 2001, p. 94, Hua XII, Beilage VI, p. 435 (trad. J. English in Articles sur la logique, Paris, PUF, 1975, p. 498). B. Russell, Introduction to Mathematical Philosophy, chapt. VII, Londres, G. Allen \& Unwin Ltd, 1921 (trad. F. Rivenc, Introduction à la philosophie mathématique, Paris, Payot, 1991, p. 149-154).

${ }^{58}$ Nous sommes largement redevable dans ce qui suit à l'excellente «Note introductive» d’Hourya Benis-Sinaceur à sa traduction de Dedekind (La création des nombres, op. cit., p. 23 sqq.). 
conquis par son moyen $»^{59}$ : s'agissant des entiers naturels, il s'agit $\mathrm{d}^{\prime}$ effectuer une construction purement ensembliste de l'ensemble des entiers en le reconduisant au concept fondamental de chaîne (Kette), c'est-à-dire d'application d'un ensemble sur l'un de ses sous-ensembles, c'est-à-dire d'effectuer une fondation ensembliste de l'arithmétique élémentaire sur le concept simple d'application (en allemand Vorstellung, représentation). De même pour la prétendue création des irrationnels : loin de postuler au cas par cas une coupure irrationnelle quand il n'en existe pas de rationnelle, la méthode de Dedekind consiste à donner une définition de l'essence de la continuité en général (c'est-à-dire valable pour tous les domaines d'idéalités, arithmétiques et géométriques : l'existence d'un et un seul élément qui effectue toute coupure entre deux classes disjointes et ordonnées d'éléments) à l'aide d'une relation d'ordre et du concept du coupure, sans aucun recours à quelque intuition extrinsèque de la continuité de la droite; son projet est de définir rigoureusement les nombres réels «sans aucune interférence de représentations étrangères » de nature géométrique ${ }^{60}$, en les reconduisant au concept antérieur de nombre rationnel ${ }^{61}$ - et ce, en une définition intensionnelle et structurale de l'ensemble des nombres réels par l'essence de la continuité, qui doit constituer « le fondement scientifique pour l'investigation de tous les domaines continus $»^{62}$.

Prêtons à présent attention à un point particulier de la démonstration de Dedekind : l'écart entre la généralité de la visée thématique et la particularité de la saisie du sens. Si son projet est de dégager l'essence de la continuité en général, elle est cependant d'abord saisie sur l'exemple de la droite ${ }^{63}$, au motif que la droite contient des points que l'on ne peut mettre en correspondance avec aucun nombre rationnel. Cela ne signifie pas que la continuité arithmétique soit conçue sur le modèle de la continuité géométrique de la droite supposée intuitivement donnée, mais que la propriété structurale de continuité, inhérente à tous les ensembles continus en général, est d'abord dégagée sur le paradigme de la droite pour être énoncée en termes purement ensemblistes (distribution des points en deux classes disjointes et

\footnotetext{
${ }^{59}$ R. Dedekind, Was sind und was sollen die Zahlen?, GMS III, trad. p. 134.

${ }^{60}$ R. Dedekind, Was sind und was sollen die Zahlen?, GMS III, trad. p. 134 ; "Sur la théorie des nombres entiers algébriques », GMS III (trad. p. 246 note).

${ }^{61}$ R. Dedekind, Was sind und was sollen die Zahlen?, GMS III, trad. p. 138 ; "Sur la théorie des nombres entiers algébriques », GMS III (trad. p. 246 note).

${ }^{62}$ R. Dedekind, Stetigkeit und irrazionale Zahlen, §3, GMS III (trad. "Continuité et nombres irrationnels ", ibid., p. 72).

${ }_{63}$ R. Dedekind, Stetigkeit und irrazionale Zahlen, § 3, GMS III (trad. ibid., p. 69 sqq.).
} 
ordonnées), avant d'être généralisée et comprise comme trait eidétique de tous les domaines continus - en particulier arithmétiques. Cela nous permet de dégager une loi générale régissant la progressivité ou l'historicité de la pensée mathématique, à savoir son fonctionnement paradigmatique: une propriété ou une structure est d'abord saisie dans son adhérence à un domaine naturel avant de devenir objet thématique d'une considération générale et abstraite.

La progressivité essentielle à la rationalité mathématique consiste précisément en cette forme de phénoménalité dans l'ordre des idéalités : une propriété ou une structure n'apparaît jamais d'emblée dans sa pureté abstraite, mais se révèle de prime abord dans un domaine d'idéalités particulier qui la possède et a, vis-à-vis de sa thématisation abstraite, la fonction de domaine d'apprentissage ; elle se manifeste d'abord comme idéalité liée ou adhérente, avant de se dévoiler comme idéalité libre. Nous reprenons ici une distinction que fait Husserl, en en modifiant le sens: les idéalités libres (freie Idealitäten) sont pour lui des objectités idéales, qui possèdent omnitemporalité (validité pour tout temps) et omnisubjectivité (validité pour quiconque) quant à leur possible réactivation et qui, bien que leur découverte ait lieu dans le monde, ne participent pas de la réalité mondaine (telles sont les objectités logico-mathématiques) ; a contrario, les idéalités liées (gebundene Idealitäten), bien que répétables de manière ubiquitaire, impliquent en leur sens d'être une réalité et une appartenance au monde réal (comme la Madone de Raphaël, qui existe à titre de chose matérielle dans l'espace, même si elle est reproductible à l'infini ${ }^{64}$. Transposons cette distinction sur le plan des seules idéalités mathématiques, en faisant cette fois abstraction de tout rapport à la réalité mondaine : une idéalité sera dite libre si elle est thématisée pour ellemême dans sa pureté et dans une totale indifférence à l'égard des domaines d'objets auxquels elle est appliquée (par exemple, la structure de groupe en général, ou la notion abstraite d'ensemble en général) ; elle sera dite liée ou adhérente si elle n'est envisagée que dans son appartenance à une pluralité d'objets idéaux donnés (par exemple, les groupes finis de permutations des racines d'une équation algébrique, ou l'ensemble des points d'une droite).

Telle est le procédé que Cavaillès appelle paradigme, dont on voit à présent qu'il possède une portée universelle, vu qu'il caractérise le mode de manifestation des idéalités mathématiques en général : elles apparaissent d'abord à l'état implicite et pré-thématique. C'est là une thèse sur laquelle Desanti a constamment porté l'accent :

${ }^{64}$ Cf. E. Husserl, Erfahrung und Urteil, § 65, p. 320-321 (trad. p. 323-324). 
Euclide ne sait pas qu'il manipule des structures. Il les utilise sans les thématiser ${ }^{65}$.

Avant de devenir elles-mêmes objets formels de la mathématique formelle, les «structures » ont été à l'œuvre dans la mathématique à ses divers degrés de formalisation et d'axiomatisation. Elles ont été maniées et connues de l'intérieur avant d'avoir été posées comme thèmes explicites d'une activité mathématique les concernant en propre $^{66}$.

[la] structure est toujours en devenir pour elle-même [...] ; dans [le] champ [opératoire] la structure est toujours en chantier : elle est domaine de possibilités pour un enchaînement ouvert d'opérations réglées. [...] toute structure n'est maniable qu'intriquée à un domaine (possible) d'idéalité ; [...] à son tour ce domaine n'est pensable que s'il enveloppe et domine les actes qui ont permis de mettre au jour l'exigence de la structure elle-même ${ }^{67}$.

[...] l'objet «fonction arbitraire» se trouve manié avant d'être pensé ${ }^{68}$.

À l'origine de toute généralisation de cet ordre se trouve, en général, un corps de théorèmes qu'on exporte hors de sa région native et qu'on travaille pour son compte, en dégageant les systèmes d'objets les plus généraux, et en formulant les hypothèses minimales capables de délimiter le champ de validité et de productivité des théorèmes considérés. De cette manière « commencent à exister » des structures qu'on ne connaissait pas, c'est-à-dire des structures qui n'étaient pas définies comme objets mathématiques explicites ${ }^{69}$.

Donnons quelques exemples de cette antériorité de l'implicite, qui constitue une structure eidétique de l'historicité de la raison.

Partant de l'ensemble $\mathbb{R}$ des nombres réels, Desanti le définit comme un ensemble de points où valent les relations d'égalité, d'appartenance, d'inclusion, d'intersection, et possédant un certain nombre de propriétés liées à la relation usuelle d'ordre, à la métrique usuelle et à l'axiome de continuité ; or, loin d'être irrémédiablement adhérentes au systèmes d'objets sur lequel elles sont définies $(\mathbb{R})$, ces relations et propriétés peuvent être dénaturées, arrachées au domaine naturel où elles valaient, pour être thématisées dans leur pureté ou "dans une indifférence d'essence à l'égard des termes qu'elle(s)

\footnotetext{
${ }^{65} \mathrm{~J}$.-T. Desanti, «La connexion des notions de genèse et de structure », in Mathesis, idéalité et historicité, Lyon, ENS Éditions, 2014, p. 149.

${ }^{66} \mathrm{Ibid} .$, p. 132.

${ }^{67}$ Ibid., p. 137.

68 J.-T. Desanti, «Sur le devenir de la science », op. cit., p. 111.

${ }^{69}$ J.-T. Desanti, «Disparitions, structures et mobilité », in La philosophie silencieuse, Paris, Seuil, 1975, p. 168.
} 
relie(nt) $\gg^{70}$, relativement à un domaine d'objets idéal où elles valent comme système de possibilités opératoires. Ainsi se laisse dégager une modalité d'engendrement historique des idéalités : l'arrachement de relations et de propriétés au domaine d'investissement primitif qui avait fourni l'occasion de leur découverte, et leur position comme pures fonctions dans un domaine opératoire purifié.

Autre exemple : la méthode cantorienne de la diagonale, destinée à montrer que l'ensemble des parties d'un ensemble a une puissance supérieure à ce dernier, pose l'existence de l'ensemble des parties sans cependant pouvoir en indiquer de procédé de construction ; l'objet ensemble des parties d'un ensemble est donc posé comme un en soi subsistant, substrat d'un horizon de propriétés ; mais la thématisation de ces propriétés ne pourra avoir lieu que dans une théorie abstraite des ensembles, de sorte que "la médiation du concept d" "ensemble abstrait" s'y exerçait, bien qu'il n'existât pas encore [...] de théorie abstraite des ensembles ${ }^{71}$. C'est dire que les idéalités explicites prises pour thèmes d'une théorie enveloppent un horizon d'idéalités implicites qui conditionne l'intelligibilité des premières, mais ne pourra être thématisé pour soi-même que dans un moment second. Le caractère second de la thématisation des idéalités implicites constitue donc un moment structurel essentiel de l'historicité des mathématiques : il est de l'essence de la manifestation de la vérité que les idéalités apparaissent d'abord à l'état naïf, investies dans un champ naturel, pour n'être ressaisies qu'ensuite dans leur pureté structurale.

\section{Conclusion}

Si les méditations husserliennes sur l'histoire de la géométrie étaient censées posséder une valeur exemplaire susceptible d'éclairer les structures eidétiques de l'histoire universelle, il nous a semblé que la relation paradigmatique était à inverser : Husserl conçoit l'histoire universelle à partir de l'histoire de la philosophie, et entend celle-ci comme un processus téléologique ouvert à l'origine par un acte $\mathrm{d}$ 'instauration originaire et orienté par une dynamis inconsciente vers la forme finale qu'est la phénoménologie transcendantale ; et ce sont ces traits essentiels qu'il transpose à l'histoire des mathématiques, pour concevoir cette dernière comme un procès téléologique initié par la constitution originelle des premières idéalités et orienté comme

\footnotetext{
70 J.-T. Desanti, Les Idéalités mathématiques, Paris, Seuil, 1968, p. 60.

${ }^{71}$ Ibid., p. 86.
} 
une marche plus ou moins consciente vers une formalisation toujours plus grande, confinant à la technicisation oublieuse de son sens. Quant à la synthèse continuelle en laquelle consiste son travail permanent, c'est un engendrement d'idéalités par des synthèses syntaxiques et polythétiques converties en actes monothétiques par nominalisation. Husserl ne ressaisit ainsi que des structures très générales, voire formelles, issues de l'auto-interprétation de la situation historique de la phénoménologie.

Dans le sillage de Cavaillès et Desanti, nous avons tâché de découvrir la logique de l'engendrement de nouveaux champs idéaux: logique anonyme dont la nécessité génératrice $\mathrm{n}^{\prime}$ est plus celle des actes producteurs du sujet pur, mais est inscrite dans la texture des champs d'idéalités. Ce qui est décisif, c'est la manière dont un domaine porte en soi une structure de manque qui appelle et fait signe vers de nouvelles thématisations; ce que nous appelons sujet se réduit à la région de manifestation de la transformation progressive des champs noématiques, à la simple intériorisation d'exigences sises au cœur de ces derniers, liées pour l'essentiel à l'écart entre l'explicite et l'implicite ainsi qu'entre l'opératoire et le thématique.

A-t-on pour autant épuisé ainsi l'interrogation sur l'essence de l'historicité ? Car il reste une question essentielle : au-delà de la logique historique de l'engendrement des idéalités, quelle est la logique qui régit la mutation des formes de mathesis, des modalités de la praxis mathématicienne? Est-elle le fait des actes instaurateurs du sujet pur? Et obéitelle à la seule loi du primat de l'implicite sur les structures qui le portent à sa pleine intelligibilité ? 